\title{
Nouvelle année, nouvel habit
}

\section{Bruno Kesseli}

Dr et lic. phil., rédacteur en chef du Bulletin des médecins suisses

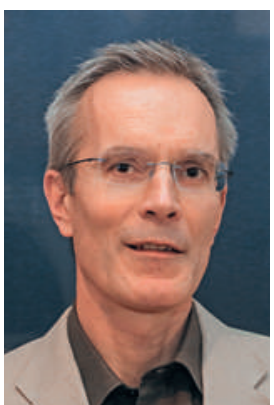

Nous n'avons certes pas réinventé la roue, mais vous avez dû tout de même, chère lectrice, cher lecteur, être surpris-e en voyant le premier BMS de cette nouvelle année. La page de couverture, déjà, se différencie nettement de l'ancienne apparence, avec la grande photo et la nouvelle présentation. En feuilletant le bulletin, l'impression se confirme: un souffle de nouveauté a apporté quelques changements visuels au vénérable «journal jaune».

Dix ans à peine après la dernière retouche de la mise en page, il nous a semblé qu'il était grand temps de procéder à un nouveau "rafraîchissement». Pas seulement pour le Bulletin des médecins suisses, mais pour toutes les revues des Editions médicales suisses EMH Media, qui affichent dorénavant une présentation unifiée et montrent clairement leurs origines communes.

«Fraîcheur»: je suis las d'entendre ce mot galvaudé dans notre maison comme une formule magique dès qu'il est question d'adaptations de la mise en page, et pourtant il décrit plutôt bien l'essence même de ce que nous avons cherché à atteindre. Même si au final, c'est surtout le contenu qui compte, la première impression est celle que donne l'«aspect extérieur». Le "design», l'emballage, envoie une multitude de signaux qui sont, en majeure partie de ma-

Même si au final, c'est surtout le contenu qui compte, la première impression est celle que donne l'«aspect extérieur».

nière inconsciente, enregistrés par les lecteurs et font naître une impression spécifique. Cette dernière ne doit pas, dans le cas des revues EMH, être associée à des notions telles que "vieux jeu" ou "poussiéreuse" mais favoriser les attentes envers des sujets d'actualité, intéressants et pertinents pour le corps médical, traités avec sérieux et de façon stimulante. Le fait que l'harmonisation vers laquelle nous tendions et qui est aujourd'hui réalisée ait posé certains défis s'explique essentiellement par l'écart stylistique important entre nos différentes revues. Les styles vont du journal scientifique aux titres comme le BMS, dont la forme se rapproche de celle des magazines grand public, en passant par les revues de formation continue. Apporter une ligne directrice commune au milieu de cette diversité, sans pour autant imposer aux différentes revues une structure trop rigide, n'était pas chose aisée. A tout cela est venue s'ajouter la nécessité économique d'utiliser avec parcimonie l'espace à disposition, c'est-à-dire de placer un nombre judicieux de caractères. Nous pensons que le compromis atteint entre les exigences fonctionnelles, esthétiques et économiques est à la hauteur des attentes. Nous espérons que vous partagerez cette opinion une fois habitués au résultat.

Chez EMH Media, nous croyons (aussi) en l'avenir de la presse écrite. Et pas par lyrisme nostalgique.

Les nouveaux médias sont sur toutes les lèvres. Nous cherchons nous aussi à suivre les évolutions actuelles, notamment en améliorant en continu la présentation Internet des revues EMH. Nous nous réjouissons d'exploiter à leur maximum les possibilités offertes pour nos revues en ligne. La nouvelle présentation de nos revues imprimées, dans laquelle nous nous sommes beaucoup investis, doit cependant éga-

lement montrer que chez EMH Media, nous continuons à croire (aussi) en l'avenir de la presse écrite. Non pas par lyrisme nostalgique, mais parce que les données des études sur les médias prouvent que le comportement de lecture du public suisse vis-à-vis des supports imprimés est resté stable ces dernières années, malgré l'évolution extrêmement rapide de la communication électronique. Le futur mot d'ordre pour les lectrices et les lecteurs du BMS pourrait donc être: encourager l'un sans délaisser l'autre. Il ne faut naturellement pas non plus oublier ce principe: à chacun, et à chacune, son support favori. Dans cet esprit, je vous souhaite, chère lectrice, cher lecteur, tous mes vœux pour la nouvelle année. 